\title{
“N" structure for type-II superlattice photodetectors
}

\author{
Omer Salihoglu, ${ }^{1}$ Abdullah Muti, ${ }_{1}^{1}$ Kutlu Kutluer, ${ }^{2}$ Tunay Tansel, ${ }^{2}$ Rasit Turan, ${ }^{2}$ \\ Yuksel Ergun, ${ }^{3}$ and Atilla Aydinli ${ }^{1, a)}$ \\ ${ }^{1}$ Department of Physics, Bilkent University, 06800 Ankara, Turkey \\ ${ }^{2}$ Department of Physics, Middle East Tzechnical University, 06531 Ankara, Turkey \\ ${ }^{3}$ Department of Physics, Anadolu University, 26470 Eskisehir, Turkey
}

(Received 26 June 2012; accepted 30 July 2012; published online 14 August 2012)

\begin{abstract}
In the quest to raise the operating temperature and improve the detectivity of type II superlattice (T2SL) photodetectors, we introduce a design approach that we call the " $\mathrm{N}$ structure." $\mathrm{N}$ structure aims to improve absorption by manipulating electron and hole wavefunctions that are spatially separated in T2SLs, increasing the absorption while decreasing the dark current. In order to engineer the wavefunctions, we introduce a thin AlSb layer between InAs and GaSb layers in the growth direction which also acts as a unipolar electron barrier. Unlike the symmetrical insertion of $\mathrm{AlSb}$ into GaSb layers, $\mathrm{N}$ design aims to exploit the shifting of the electron and hole wavefunctions under reverse bias. With cutoff wavelength of $4.3 \mu \mathrm{m}$ at $77 \mathrm{~K}$, temperature dependent dark current and detectivity measurements show that the dark current density is $3.6 \times 10^{-9} \mathrm{~A} / \mathrm{cm}^{2}$, under zero bias. Photodetector reaches background limited infrared photodetection (BLIP) condition at $125 \mathrm{~K}$ with the BLIP detectivity ( $\mathrm{D}^{*}{ }_{\text {BLIP }}$ ) of $2.6 \times 10^{10}$ Jones under $300 \mathrm{~K}$ background and $-0.3 \mathrm{~V}$ bias voltage. (C) 2012 American Institute of Physics. [http://dx.doi.org/10.1063/1.4745841]
\end{abstract}

Type II superlattice (T2SL) InAs/GaSb photodetectors have received great interest in the development of short wave infrared (SWIR), midwave infrared (MWIR), and long wave infrared (LWIR) detectors due to advantages like band gap engineering, ${ }^{1}$ suppression of Auger recombination, ${ }^{2}$ strain induced splitting of heavy hole and light hole bands, and reduced interband tunneling due to higher effective masses of electrons and holes. ${ }^{3}$ Despite different performance levels, recent demonstration of SWIR, MWIR, and LWIR operation shows the flexibility of the superlattice material system ${ }^{4}$ opening the way multiple band detectors. MWIR InAs/GaSb superlattice photodiode focal plane arrays (FPAs) operating at $77 \mathrm{~K}$ have already been demonstrated. ${ }^{5}$ However, considering the range of advantages offered by it, the goal of operating at higher temperatures with high quantum efficiencies remains. Fundamental stumbling block to high temperature operation is the relatively low optical absorption and increasing dark current. Several proposals have been made to overcome these problems, introduction of energy barriers being the most promising. To this end combination of bulk and superlattice barriers with superlattice absorbers are being intensively studied. ${ }^{6-11}$ Lattice matching using appropriate material combinations with favorable conduction band offsets for both electrons and holes is possible. A unipolar barrier with band gap larger than that of the absorber region reduces diffusion currents while maintaining the photocurrent level. Barriers with and without superlattice structures have been utilized in $\mathrm{nBn}$ design, ${ }^{6} \mathrm{PbIbN}$ design, ${ }^{7}$ and CBIRD structures. ${ }^{8}$ Klipstein et al. demonstrated an $\mathrm{XBn}$ design reaching blip temperature of $160^{\circ} \mathrm{C}$ and quantum efficiency of $70 \%$ with cutoff wavelength of $4.1 \mu \mathrm{m} .{ }^{9}$ Further, complex supercells containing designs like "W" structure ${ }^{10}$ and " $\mathrm{M}$ " structure ${ }^{11}$ have been introduced as

\footnotetext{
${ }^{\text {a) }}$ Author to whom correspondence should be addressed. Electronic mail: aydinli@fen.bilkent.edu.tr. Tel.: +90 (312) 2901579.
}

bipolar barriers with various degrees of performance. Besides reducing the dark current and increasing the differential resistance-area product, "M" design also aims to increase the overlap integral between electron and hole wavefuntions, intending to attain higher optical absorption. It has been shown that $\mathrm{M}$ design has positive effect on electron-hole overlap which enhances the optical properties of the material.

In this letter, we introduce a unipolar barrier complex supercell superlattice system which increases electron-hole overlap under bias, significantly. Named as "N structure," it is similar to a superlattice pin diode, but in contrast with the symmetrical $\mathrm{M}$ design, where AlSb is inserted in the middle of the GaSb layer, it has two monolayers (MLs) of AlSb inserted asymmetrically between InAs and GaSb layers, along the growth direction, as an electron barrier. It is well known that electron and hole wavefunctions shift under bias due to the tilting of the energy band diagram. In a symmetrical barrier design under bias, the electron-hole wavefunction overlap increases on one side of the barrier while it decreases on the other due to directionality of the electric field. In the case of an asymmetrical barrier placed with the direction of the bias field in mind, absorption increases without any loss of overlap. Despite the difficulty of perfect lattice matching of InAs and AlSb, such a design is expected to reduce dark current significantly, as in the M case.

Conduction and valance band profiles for the proposed T2SL "N" structure is shown in Fig. 1(a). The design is named $\mathrm{N}$ structure since the lineup of the successive materials gives the impression of the capital letter "N." Under bias, in a standard pin diode, electron and hole wavefunctions shift in opposite directions. Inserting an AlSb layer symmetrically into the middle of the GaSb layer leads to an increase of the overlap at the InAs/GaSb interface on the lower energy side of the AlSb barrier, while it becomes smaller at the InAs/GaSb interface on the higher energy side. However, 
(a)

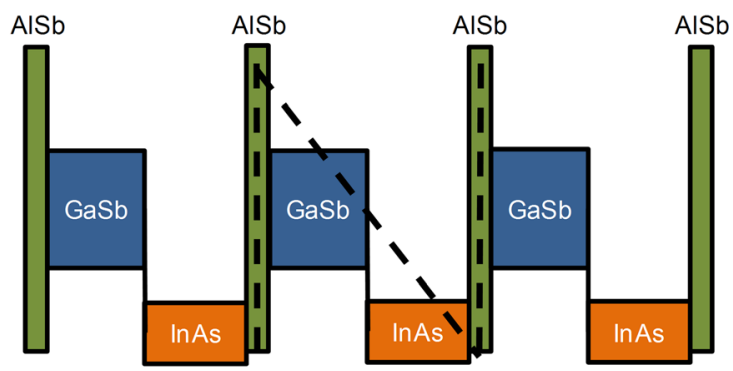

(b)

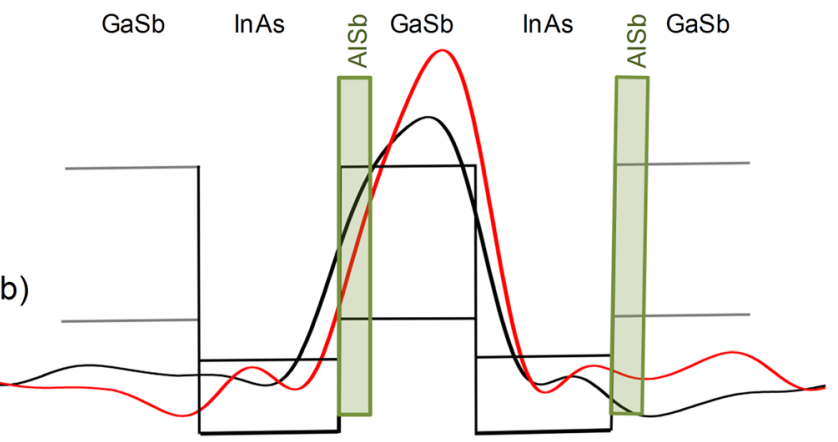

FIG. 1. (a) Conduction and valance band profiles for the proposed T2SL "N" structure. (b) Overlap integral with (red line) and without (black line) AlSb barrier under $0.001 \mathrm{meV}$ bias. Negative bias is applied to the right side of the structure.

when the AlSb barrier is introduced between $\mathrm{GaSb}$ and InAs layers, electron and hole wavefunctions are pushed away from the AlSb interface, increasing the overlap at the InAs/ $\mathrm{GaSb}$ interface. Figure 1(b) shows the overlap integral with and without AlSb barrier under $0.001 \mathrm{meV}$ bias per period. When there is no barrier (black line), overlap integral is shifted towards right side of the GaSb layer. With the AlSb barrier, (red line) the shift of the overlap integral towards the $\mathrm{InAs} / \mathrm{GaSb}$ interface is enhanced. It is clear that barrier increases the overlap at the GaSb/InAs interface. Our calculations of electron-hole wavefunction overlap integral show that the absorption at the interface increases by about $\% 25$ when an AlSb barrier is used. The barrier is expected to increase optical absorption and as well as lower the diffusion current by blocking the thermally generated electrons. AlSb barrier blocks the interaction between two consecutive pairs, therefore, also should reduce the tunneling probability and increase the electron effective mass.

The detector is designed as p-i-n photodetector. It starts with $100 \mathrm{~nm}$ thick $\mathrm{GaSb}$ buffer layer and $20 \mathrm{~nm} \mathrm{Al}(\mathrm{x}) \mathrm{Ga}$ $\mathrm{As}_{(\mathrm{y})} \mathrm{Sb}$ as an insulator and etch stop layer, followed by $1000 \mathrm{~nm} \mathrm{GaSb}: \mathrm{Be}\left(\mathrm{p}=1.0 \times 10^{17} \mathrm{~cm}^{-3}\right) \mathrm{p}$ contact layer. P-i-n part of the design consist of 90 periods 9 MLs of InAs/2 MLs of AlSb/8.5 MLs of GaSb:Be $\left(p=1.5 \times 10^{17} \mathrm{~cm}^{-3}\right)$ p-type layers, 60 periods 9 MLs of InAs/2 MLs of AlSb/8.5 MLs of GaSb i-layers, 40 periods 9 MLs of InAs:Te (n: $5 \times 10^{17} \mathrm{~cm}^{-3}$ )/2 MLs of AlSb/8.5 MLs of GaSb n-type layers and structure is terminated by $20 \mathrm{~nm}$ InAs:Te (n: $5 \times 10^{17} \mathrm{~cm}^{-3}$ ) cap layer to assure good ohmic contact. The sample studied in this work was grown commercially (IQE Inc. USA) with molecular beam epitaxy on a GaSb substrate. High resolution $\mathrm{x}$-ray diffraction (HRXRD) spectrum is shown in Figure 2. The periodicity of the superlattice as well as the mismatch of the zeroth order superlattice diffraction peak to the underlying GaSb substrate were determined using symmetric (004) x-ray diffraction peak. Narrow FWHM of the peaks and higher order diffraction represents perfect crystalline quality and uniform superlattice periods at the grown epilayer. A linear least squares fit of the peak spacing was used to determine the periodicity of the structure as $67.67 \AA$. The mismatch of the zeroth order peak relative to the GaSb substrate is determined as $1566 \mathrm{ppm}$. This mismatch can be attributed to not having common atoms between AlSb and InAs layers and is very close to almost zero lattice mismatch limit $(<1000 \mathrm{ppm})$.

Single pixel photodetectors were fabricated with mesa sizes ranging from $100 \times 100$ to $700 \times 700 \mu \mathrm{m}$. To minimize surface damage, mesas have been fabricated by standard lithography and phosphoric acid based wet etch solution. The etch process has been stopped when etch depth reached the bottom contact layer. The complete fabrication processes can be found elsewhere. ${ }^{12}$ A protective $250 \mathrm{~nm}$ thick $\mathrm{SiO}_{2}$ layer has been deposited using plasma enhanced chemical vapor deposition (PECVD) system at $160^{\circ} \mathrm{C}$ with $\% 2 \mathrm{SiH}_{4} /$ $\mathrm{N}_{2}$ and $\mathrm{N}_{2} \mathrm{O}$ gas flows of $180 \mathrm{sccm}$ and $225 \mathrm{sccm}$, respectively. Ohmic contacts were made by evaporating $5 \mathrm{~nm}$ titanium (Ti) and $200 \mathrm{~nm}$ gold ( $\mathrm{Au}$ ) on the bottom and top contact layers of the detectors. Contact resistances have been measured as $2.5 \Omega$ and $7.2 \Omega$ for the n- and p-type contacts, respectively. Sample was bonded to a chip carrier for further characterization.

Electrical measurements of the " $\mathrm{N}$ " design superlattice barrier structure have been done by a HP4142OA sourcemeasure unit. Samples were mounted on a He cooled closed cycle cold finger with a cold shield system. Dark current measurements were performed at temperatures between $30 \mathrm{~K}$ and $250 \mathrm{~K}$. Figure 3 shows the measured dark current density vs applied bias voltage characteristics of the $700 \mu \mathrm{m}^{2}$ diodes, chosen to ensure that the surface leakage does not dominate for different temperatures. At $77 \mathrm{~K}$ and under $-0.1 \mathrm{~V}$ bias voltage, the dark current density is measured as $5.5 \times 10^{-7} \mathrm{~A} / \mathrm{cm}^{2}$ and corresponding dynamic resistancearea product (RA) is determined as $1.6 \times 10^{5} \Omega \mathrm{cm}^{2}$. Even at

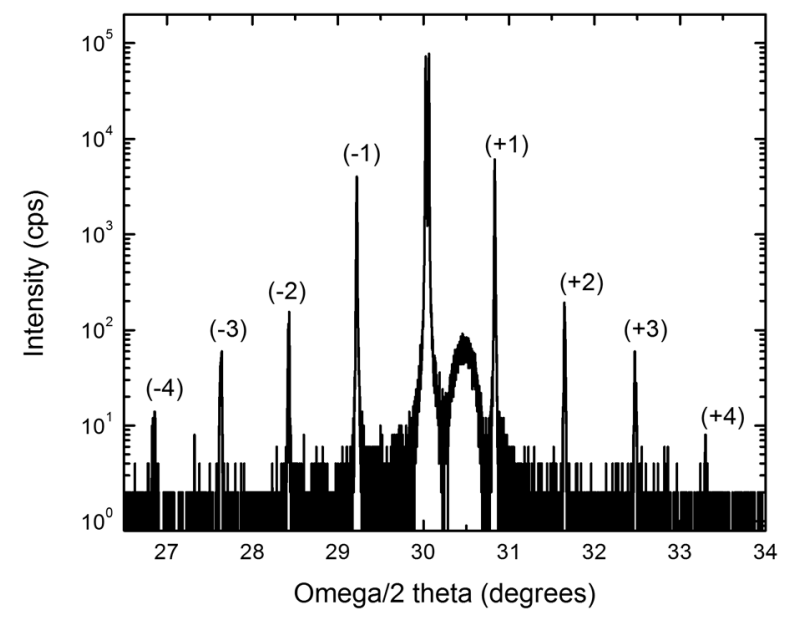

FIG. 2. High resolution x-ray diffraction curves of the superlattice along the 004 direction. Narrow FWHM of the peaks and highly ordered diffraction indicates uniform superlattice periods throught the grown epilayer and excellent crystalline quality. 


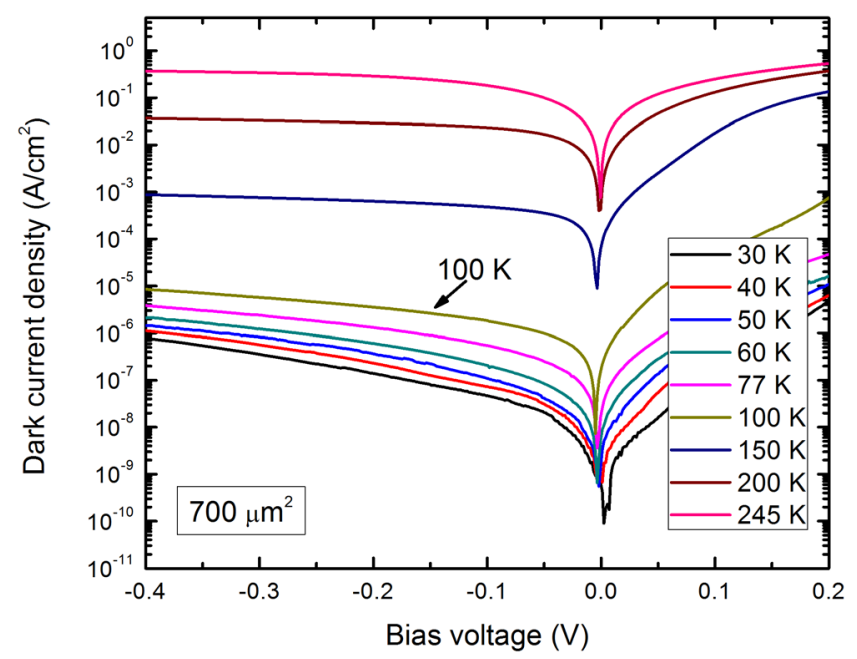

FIG. 3. Dark current density vs applied bias voltage characteristics of the $700 \mu \mathrm{m}^{2}$ diodes for different temperatures.

$150 \mathrm{~K}$, and under $-0.1 \mathrm{~V}$ bias, dark current density and RA values are determined as $4.8 \times 10^{-4} \mathrm{~A} / \mathrm{cm}^{2}$ and $0.6 \times 10^{3} \Omega \mathrm{cm}^{2}$, respectively.

Figure 4(a) shows dark current density and dynamic resistance area product vs. 1000/T graph for $700 \mu \mathrm{m}^{2}$ photodiode for different temperatures under zero bias. The I-V
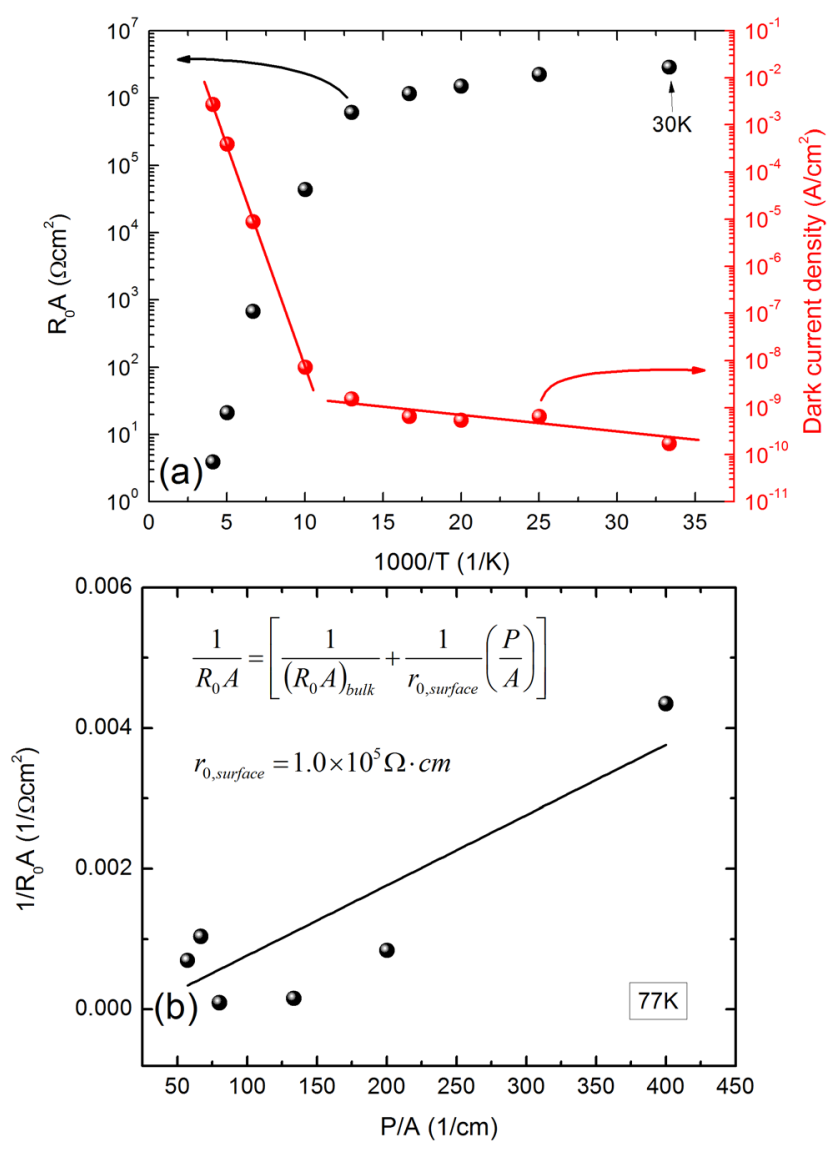

FIG. 4. (a) Temperature dependent dark current density for type-II InAs/ GaSb superlattice $700 \times 700 \mu \mathrm{m}$ photodiodes at zero bias voltage. Two linear fits show diffusion limited region for high temperatures and generationrecombination (G-R) limited region for low temperatures. (b) Dynamic resistance-area product at zero bias vs. perimeter to area ratio at $77 \mathrm{~K}$. curve is dominated by diffusion current for temperatures higher than $77 \mathrm{~K}$ and by Generation recombination (G-R) current for temperatures lower than $77 \mathrm{~K}$. For higher temperatures, Arhenious type behavior has been observed. The activation energy has been determined as $0.261 \mathrm{eV}$ at $0.2 \mathrm{~V}$ which is very close to material bandgap. At $77 \mathrm{~K}$, dark current density and dynamic resistance at zero bias are measured as $1.5 \times 10^{-9} \mathrm{~A} / \mathrm{cm}^{2}$ and $6.1 \times 10^{5} \Omega \mathrm{cm}^{2}$, respectively. This very low dark current density shows that lattice mismatch of the InAs and AlSb does not cause a dramatic defective region at the interface. At $150 \mathrm{~K}$, dark current density and dynamic resistance at zero bias are measured as $1.5 \times 10^{-6} \mathrm{~A} / \mathrm{cm}^{2}$ and $6.8 \times 10^{2} \Omega \mathrm{cm}^{2}$, respectively. These results are very promising for high temperature operation. This low dark current density satisfies the requirements of the commercially available read out integrated circuits (ROICs) for the operation at $150 \mathrm{~K}$. The inverse dynamic resistance area product at zero bias as a function of the perimeter to area ratio at $77 \mathrm{~K}$ is shown in Figure 4(b). The surface part of the resistance-area product can be calculated from the slope of the graph. Calculated surface resistivity $\left(\mathrm{r}_{\text {surface }}\right)$ is $1.0 \times 10^{5} \Omega \mathrm{cm}$. This relatively high surface resistivity shows suppressed surface related currents due to the $\mathrm{N}$ barrier design. Placing the electron barrier is known to impede the electron flow, leading to reduction of the surface leakage. ${ }^{13,14}$ In addition, it also is expected to reduce band to band and trap assisted tunneling currents. ${ }^{13}$

Spectral response of the photodetectors has been measured using calibrated blackbody source at $450{ }^{\circ} \mathrm{C}$ (Newport, Oriel 67000), lock-in amplifier (SRS, SR830 DSP), and mechanical chopper (SRS, SR540) system. Details of the measurement can be found elsewhere. ${ }^{15}$ For single pass front illumination condition highest quantum efficiency (QE) of the photodetector has been determined as $15 \%$ around wavelength of $3.5 \mu \mathrm{m}$. Quantum efficiency is directly related with the thickness of the absorbing region and will increase with thicker absorbing region. The cut-off wavelength of the device is determined to be $4.3 \mu \mathrm{m}$ at $77 \mathrm{~K}$. The cutoff wavelength increases to $4.9 \mu \mathrm{m}$ at $255 \mathrm{~K}$, as expected. Figure 5(a) shows Johnson-noise limited detectivity $\left(D^{*}\right)$ versus wavelength graph for the operating temperatures between $77 \mathrm{~K}$ and $250 \mathrm{~K}$. Under $-0.3 \mathrm{~V}$ bias, the peak $\mathrm{D}^{*}$, was equal to $2.9 \times 10^{12}$ Jones for the photodetector at $4.0 \mu \mathrm{m}$ and $77 \mathrm{~K}$. Detectivity at $180 \mathrm{~K}$ is $2 \times 10^{10}$ Jones. Figure $5(\mathrm{~b})$ shows $\mathrm{D}^{*}$ BLIP as a function of temperature at $300 \mathrm{~K}$ background for $\mathrm{f} / 2$ optics with the $-0.3 \mathrm{~V}$ bias voltage. Photodetector reaches BLIP condition at $125 \mathrm{~K}$ with the BLIP detectivity $\left(\mathrm{D}^{*}\right.$ BLIP) of $2.6 \times 10^{10}$ Jones under $300 \mathrm{~K}$ background and $-0.3 \mathrm{~V}$ bias voltage. These results makes $\mathrm{N}$ designed T2SL photodetectors very promising structures, for the high operating temperature (HOT) regime.

In conclusion, we have demonstrated $\mathrm{N}$ structure complex super cell design for MWIR region which showed very high $\mathrm{R}_{0} \mathrm{~A}$ and detectivity with cutoff wavelength at $4.3 \mu \mathrm{m}$ (MWIR). $\mathrm{N}$ structured superlattice photodetectors gave dark current from $5.5 \times 10^{-7} \mathrm{~A} / \mathrm{cm}^{2}$ at $77 \mathrm{~K}$ and under $-0.1 \mathrm{~V}$ applied bias condition. Corresponding RA was $1.6 \times 10^{5} \Omega \mathrm{cm}^{2}$. Detectivity (D*) is determined as $2.9 \times 10^{12}$ Jones photodetector at $4 \mu \mathrm{m}$ and $77 \mathrm{~K}$. QE of the photodetector has been determined as $15 \%$ for single pass front illumination condition. Thicker absorption 

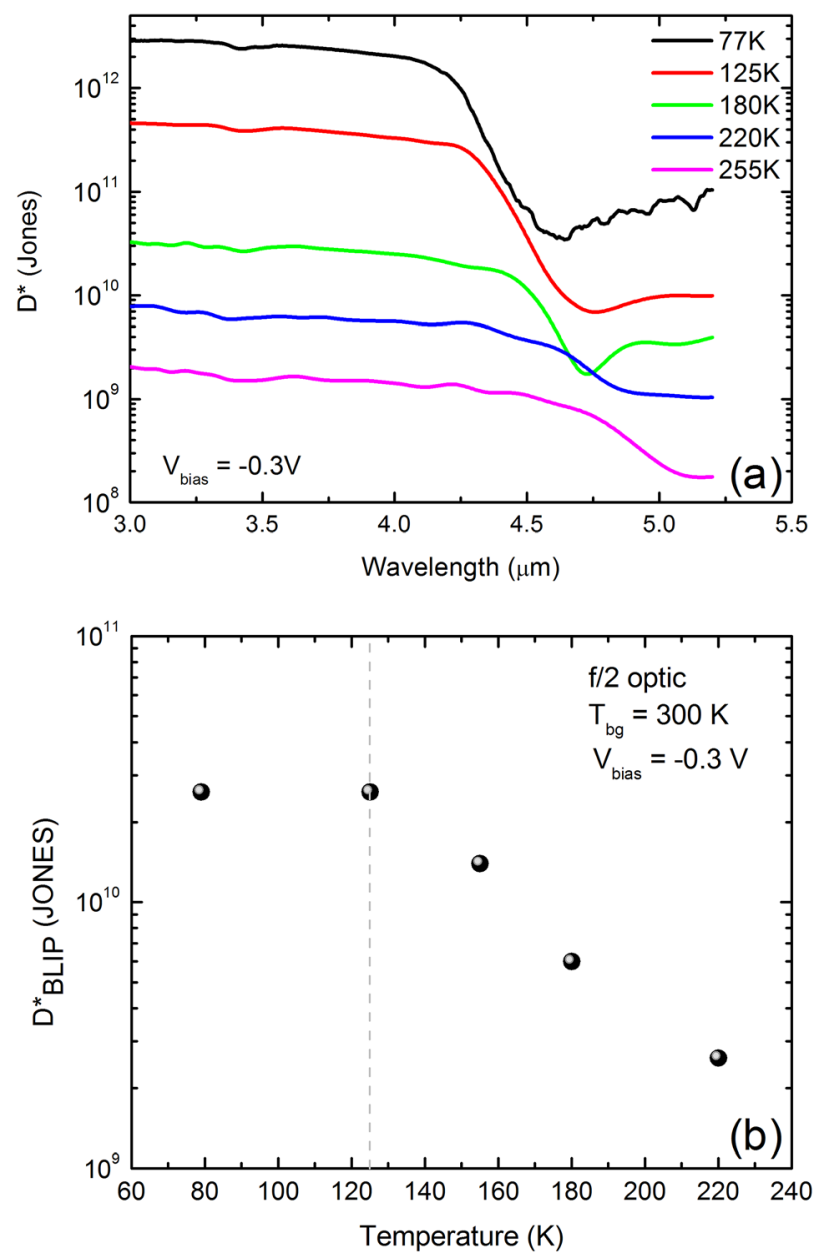

FIG. 5. (a) Johnson-noise limited detectivity ( $\mathrm{D}^{*}$ ) vs wavelength graph for the operating temperatures between $77 \mathrm{~K}$ and $250 \mathrm{~K}$ under bias voltage of $-0.3 \mathrm{~V}$. (b) $\mathrm{D}^{*}$ as a function of temperature at $300 \mathrm{~K}$ background for $\mathrm{f} / 2$ optics with the $-0.3 \mathrm{~V}$ bias voltage.

regions will increase this quantum efficiency. Temperature dependent dark current measurements revealed that dominant current is bulk diffusion current for the temperatures higher than $77 \mathrm{~K}$ and it has Arrhenius type of behavior. For the temperatures lower than $77 \mathrm{~K}$, G-R current dominates the behavior. Dynamic resistance-area product as a perimeter to area ratio measurements showed that surface resistivity $\left(\mathrm{r}_{\text {surface }}\right)$ is equal to $1.0 \times 10^{5} \Omega \mathrm{cm}$. It is important to evaluate these results in the light of lattice mismatch between InAs and AlSb, which is an order of magnitude larger than that of AlSb on GaSb. One would assume that lattice mismatch will cause trap states between InAs and AlSb interface causing high dark currents. However, since AlSb is only two monolayers thick and below the critical thickness, the tetragonally distorted interface does not seem to be generating large amount of electrically active interface states. Indeed, it is well known that InSb-like bonding at the interface can lead to high quality interfaces with good interface electronic properties. ${ }^{16}$ Our results seem to be parallel to these observations indicating that the lattice mismatch is not large enough to degrade device performance, significantly. High temperature $(150 \mathrm{~K})$ dark current density is measured as $1.5 \times 10^{-6} \mathrm{~A} / \mathrm{cm}^{2}$ at zero bias. Photodetector reaches BLIP condition at $125 \mathrm{~K}$ with the BLIP detectivity $\left(\mathrm{D}^{*}{ }_{\text {BLIP }}\right.$ ) of $2.6 \times 10^{10}$ Jones under $300 \mathrm{~K}$ background and $-0.3 \mathrm{~V}$ bias voltage. Even though direct comparison with similar structures is not possible due to subtle differences between the designs, both the RA and current density values are comparable with MWIR superlattice structures in which AISb is placed symmetrically into GaSb. Lower quantum efficiency of our samples is due to much thinner absorber layer used in our design, ultimately lowering the detectivity as well. Quantum efficiency and the detectivity of the $\mathrm{N}$ structure detector are very promising when we compare them with very recently published similar barrier type photodetectors. ${ }^{17}$ "N" structure SL photodetectors should perform much better when designed to operate in the LWIR region due to relatively small band gap of the LWIR photodetectors, where generation recombination (G-R) and surface leakage currents become dominant.

${ }^{1}$ Y. J. Wei and M. Razeghi, Phys. Rev. B 69, 085316 (2004).

${ }^{2}$ C. H. Grein, P. M. Young, and H. Ehrenreich, Appl. Phys. Lett. 61, 29052907 (1992).

${ }^{3}$ D. L. Smith and C. Mailhiot, J. Appl. Phys. 62, 2545-2548 (1987).

${ }^{4}$ M. Razeghi and S. A. Pour, Proc. SPIE 8353, 835310 (2012).

${ }^{5}$ R. Rehm, M. Walther, J. Schmitz, J. Fleissner, J. Ziegler, W. Cabanski, and R. Breiter, Electron. Lett. 42, 577-578 (2006).

${ }^{6}$ J. B. Rodriguez, E. Plis, G. Bishop, Y. D. Sharma, H. Kim, L. R. Dawson, and S. Krishna, Appl. Phys. Lett. 91, 043514 (2007).

${ }^{7}$ N. Gautam, H. S. Kim, M. N. Kutty, E. Plis, L. R. Dawson, and S. Krishna, Appl. Phys. Lett. 96, 231107 (2010).

${ }^{8}$ D. Z. Y. Ting, C. J. Hill, A. Soibel, S. A. Keo, J. M. Mumolo, J. Nguyen, and S. D. Gunapala, Appl. Phys. Lett. 95, 023508 (2009).

${ }^{9}$ P. Klipstein, O. Klin, S. Grossman, N. Snapi, I. Lukomsky, D. Aronov, M. Yassen, A. Glozman, T. Fishman, E. Berkowicz, O. Magen, I. Shtrichman, and E. Weiss, Opt. Eng. 50, 061002 (2011).

${ }^{10}$ C. L. Canedy, E. H. Aifer, I. Vurgaftman, J. G. Tischler, J. R. Meyer, J. H. Warner, and E. M. Jackson, J. Electron. Mater. 36, 852-856 (2007).

${ }^{11}$ P. Y. Delaunay, B. M. Nguyen, D. Hoffman, E. K. W. Huang, and M. Razeghi, IEEE J. Quantum Electron. 45, 157-162 (2009).

${ }^{12}$ O. Salihoglu, A. Muti, K. Kutluer, T. Tansel, R. Turan, and A. Aydinli, J. Appl. Phys. 111, 074509 (2012).

${ }^{13}$ G. R. Savich, J. R. Pedrazzani, D. E. Sidor, S. Maimon, and G. W. Wicks, Appl. Phys. Lett. 99, 121112 (2011).

${ }^{14}$ S. Maimon and G. W. Wicks, Appl. Phys. Lett. 89, 151109 (2006).

${ }^{15}$ T. Tansel, K. Kutluer, O. Salihoglu, A. Aydinli, B. Aslan, B. Arikan, M. C. Kilinc, Y. Ergun, Serincan, and R. Turan, IEEE Photonics Technol. Lett. 24, 790-792 (2012).

${ }^{16}$ J. Spitzer, A. Hopner, M. Kuball, M. Cardona, B. Jenichen, H. Neuroth, B. Brar, and H. Kroemer, J. Appl. Phys. 77, 811-820 (1995).

${ }^{17}$ G. Chen, B. M. Nguyen, A. M. Hoang, E. K. Huang, S. R. Darvish, and M. Razeghi, Appl. Phys. Lett. 99, 183503 (2011). 